\title{
Extensions to LTE Mobility Functions for ns-3
}

\author{
Budiarto Herman * \\ Magister Solutions \\ Jyväskylä, Finland \\ budiarto.herman@magister.fi
}

\author{
Nicola Baldo \\ Centre Tecnològic de \\ Telecomunicacions de \\ Catalunya \\ Barcelona, Spain \\ nicola.baldo@cttc.es
}

\author{
Marco Miozzo \\ Centre Tecnològic de \\ Telecomunicacions de \\ Catalunya \\ Barcelona, Spain \\ marco.miozzo@cttc.es
}

\author{
Manuel Requena \\ Centre Tecnològic de \\ Telecomunicacions de \\ Catalunya \\ Barcelona, Spain \\ manuel.requena@cttc.es
}

\author{
Jaime Ferragut \\ Centre Tecnològic de \\ Telecomunicacions de \\ Catalunya \\ Barcelona, Spain \\ jaime.ferragut@cttc.es
}

\begin{abstract}
ns-3 is an open-source network simulator with support for simulating 3GPP LTE cellular network. This paper presents three extensions to the LTE module of ns-3, enhancing its capability of simulating scenarios with more sophisticated user mobility requirements. The first extension implements additional features in the UE measurements function. The second extension delivers additional handover algorithms and a modular framework for ns-3 users to incorporate their own handover algorithms. At last, the third extension enables automatic network attachment using the initial cell selection procedure. The modelling of these extensions refers to $3 \mathrm{GPP}$ standard specifications. The resulting models have been validated using the ns-3 testing framework.
\end{abstract}

\section{Categories and Subject Descriptors}

1.6.5 [Simulation and Modeling]: Model DevelopmentModeling methodologies; 1.6 .7 [Simulation and Modeling]: Simulation Support System-Environments

\section{Keywords}

Handover, LTE, mobility, ns-3, Simulator

\section{INTRODUCTION}

Long Term Evolution (LTE) is the fourth generation mobile telecommunication standard, which has recently gaining traction in many parts of the world. Compared to the

\footnotetext{
${ }^{*}$ The work described in this paper was conducted when the author was an active student of University of Jyväskylä, Finland.
}

Permission to make digital or hard copies of all or part of this work for personal or classroom use is granted without fee provided that copies are not made or distributed for profit or commercial advantage and that copies bear this notice and the full citation on the first page. Copyrights for components of this work owned by others than the author(s) must be honored. Abstracting with credit is permitted. To copy otherwise, or republish, to post on servers or to redistribute to lists, requires prior specific permission and/or a fee. Request permissions from permissions@acm.org.

WNS3, May 07 2014, Atlanta, GA, USA.

Copyright ACM 978-1-4503-3003-9/14/05 ...\$15.00.

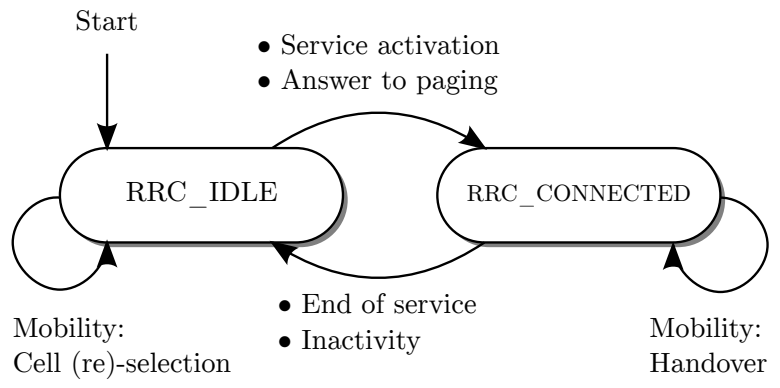

Figure 1: UE RRC states in 3GPP LTE [16]

previous generations, LTE delivers significant improvements in many aspects, such as in data rates, spectral efficiency, network architecture, and self-organising capabilities. The $3^{\text {rd }}$ Generation Partnership Project (3GPP) has been the organisation responsible for the standardisation of LTE.

The LTE cellular network is a distributed network of nodes called Evolved NodeBs (eNodeB). Each eNodeB provides network connectivity and services to the users within a certain area referred to as the cell. In an equivalent way, one can say that the User Equipments (UE) are attached to the cell, while at the same time the cell is taking the role as the serving cell of those UEs.

An important feature of an LTE system is user mobil$i t y$, which enables users to freely roam the network across different cells while experiencing minimal impact to connectivity and performance. For instance, 3GPP has specified that connectivity shall be maintained for users moving with speed up to $350 \mathrm{~km} / \mathrm{h}$ (or up to $500 \mathrm{~km} / \mathrm{h}$ in some frequency bands) [2]. LTE system is equipped with a set of procedures to achieve this target. The procedure used depends on the RRC state of the UE, which is either RRC_CONNECTED or RRC_IDLE (henceforth referred to as the Connected mode and the Idle mode) [5]. In Connected mode, user mobility is handled by the handover procedure, while in Idle mode user mobility is handled by cell selection and cell re-selection procedures, as depicted in Figure 1.

This paper looks into modelling LTE user mobility in the $n s-3$ simulator. The overall goal of this paper is to enable 
students and researchers from both academia and industry to conduct thorough study on LTE user mobility using a free and open-source network simulator such as ns-3. ${ }^{1}$

A prior work [9], included in the ns-3.18 release, addressed the need for simulating LTE user mobility using ns-3. The work has contributed a working model of X2-based handover procedure, complete with the models of interfaces, protocols, and signalling required by the corresponding $3 \mathrm{GPP}$ standard specifications. However, the resulting models still incorporated several limitations. Firstly, the process of triggering a handover was restricted to a pre-defined handover algorithm, making it difficult for ns-3 users to use a different type of handover algorithm in their simulation. Secondly, the UE measurements function had very limited configuration options, e.g., supporting only two types of trigger. Some parameters of measurements were also not available for use, such as hysteresis and time-to-trigger. Thirdly, the model did not support user mobility in Idle mode. For instance, the process of attaching a UE to the network had been done manually by the simulation script, i.e., by providing an explicit pointer to the specific eNodeB that the UE shall attach to. In a real-life LTE system, network attachment is done autonomously by the UE using the Idle mode cell selection procedure.

In this paper, we propose a set of extensions to ns-3.18 to address the above limitations. They are organised into three objectives, as described in the following:

Objective 1. Additional reporting triggers and parameters for UE measurements.

Objective 2. Modular and interchangeable handover algorithms.

Objective 3. Support for automatic network attachment by initial cell selection.

We note that several common constraints were present in the LTE module of ns-3.18. For instance, the module was strictly intra-LTE, intra-frequency, and without any support for LTE-Advanced carrier aggregation. In addition, the module had minimal support for Idle mode. This paper does not aim to overcome these limitations.

The rest of the paper is arranged as follows. Section 2, Section 3, and Section 4 are dedicated to Objective 1, 2, and 3, respectively. After that, Section 5 is dedicated to the validation of the resulting models by taking advantage of ns-3 testing framework. Finally, Section 6 concludes the paper.

\section{UE MEASUREMENTS}

In 3GPP LTE, measurement reports from the UE are the principal input to the intra-LTE intra-frequency X2-based handover procedure [5]. The UE measurements function produces these measurement reports and then submits them to the serving eNodeB. This section describes the extension to the existing ns-3 UE measurements function.

\footnotetext{
${ }^{1}$ One shall not confuse the term "user mobility" used throughout this paper with the term "mobility model" used within ns-3. The latter refers to the simulation model that maintains and manipulates the position and velocity of objects in an ns-3 simulation.
}

Table 1: Measurement report triggering events supported by the extended UE measurements function

\begin{tabular}{|l|l|}
\hline ID & DESCRIPTION \\
\hline A1 & Serving cell becomes better than threshold \\
\hline A2 & Serving cell becomes worse than threshold \\
\hline A3 & $\begin{array}{l}\text { Neighbour cell becomes offset better than serving } \\
\text { cell }\end{array}$ \\
\hline A4 & Neighbour cell becomes better than threshold \\
\hline A5 & $\begin{array}{l}\text { Serving cell becomes worse than threshold1 and } \\
\text { neighbour cell becomes better than threshold2 }\end{array}$ \\
\hline
\end{tabular}

\subsection{Extending the Configurability}

The preceding UE measurements function in ns-3.18 was restricted to two pre-defined reporting triggers. These triggers were Event A2 and Event A4, and both used Reference Signal Received Quality (RSRQ) measurements [4] as the trigger quantity.

The extension has provided the possibility to customise this trigger configuration, such as by enabling the Reference Signal Received Power (RSRP) [4] trigger quantity. In contrast with RSRQ which represents signal quality, RSRP represents signal strength.

Moreover, the extension has added the support for other reporting triggers, namely Event A1, A3, and A5. Table 1 shows the list of supported triggers in the resulting UE measurements function. The event description refers to the active trigger quantity, i.e., either RSRP or RSRQ. For more details on these event-based triggers, readers are referred to the corresponding 3GPP standard document [7].

The extension has also removed the limitation on the report interval parameter. Previously in ns-3.18, the parameter was hardcoded to $480 \mathrm{~ms}$. The extension has added the implementation of the remaining report intervals, as specified by $3 \mathrm{GPP}$ [7], resulting to 13 possible options ranging from $120 \mathrm{~ms}$ to 60 minutes.

Lastly, the extension has enabled the possibility to configure the hysteresis and time-to-trigger parameters. These parameters are useful to induce delay to reporting. Hysteresis is an offset of the strength or quality of the measurements, defined in decibels of RSRP or RSRQ, while time-to-trigger is an offset in the term of time. The handover algorithm in Subsection 3.3 demonstrates the use of these parameters.

\subsection{Consumers of UE Measurements}

Here, we introduce the term consumer of UE measurements. The term refers to a modular ns-3 simulation model which requires measurement reports from UEs to achieve its specific goal. A handover algorithm is an example of model that belongs to this category.

In order to fulfil its needs for measurement reports, each consumer instance interacts with an eNodeB RRC instance. The first interface is measurement configuration, where the consumer instance requests specific settings on what are measured and when they are reported. The eNodeB RRC instance would then forward it to the attached UEs via the $\mathrm{RRC}$ connection re-configuration procedure [7]. The second interface is measurement reporting, which is basically the eNodeB RRC instance receiving measurement reports from the UEs and forwarding them up to the interested consumer instances. In Figure 2, measurement configuration is depicted 


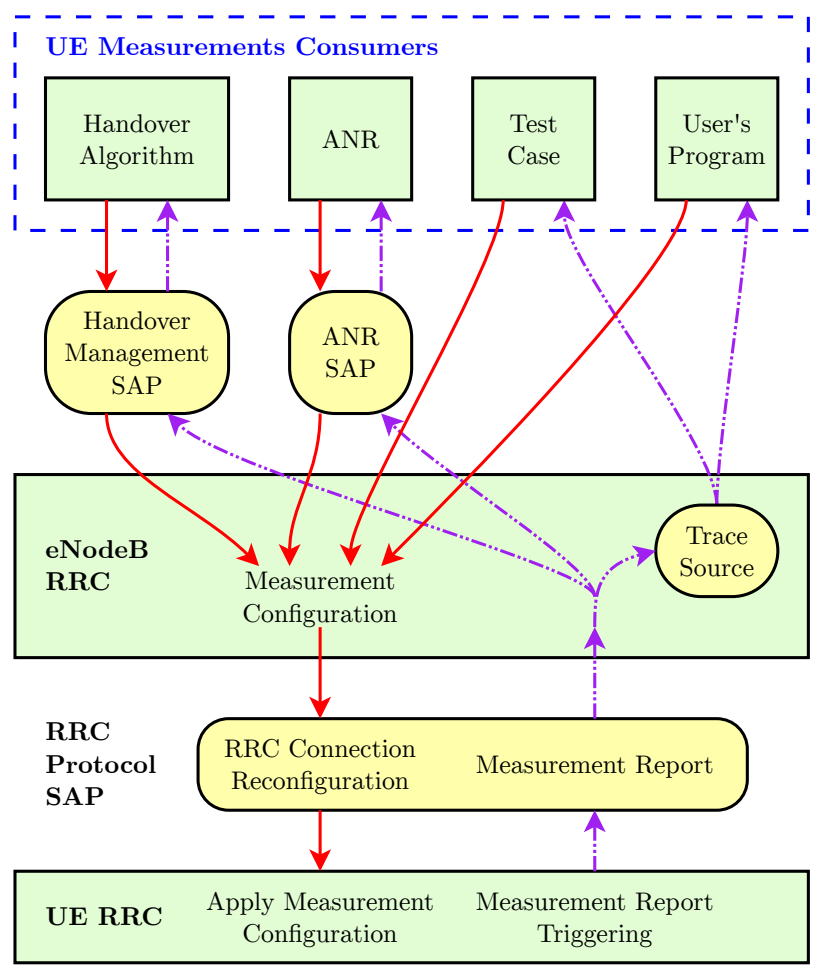

Figure 2: Relationship between UE measurements function and its consumers

by downward red-coloured arrows, while measurement reporting is depicted by upward purple-coloured arrows.

The figure also depicts the handover algorithm and the Automatic Neighbour Relation (ANR) functions as examples of internal functions which act as consumers. The figure also shows that external functions, such as test cases and user's simulation programme, are also allowed to become consumers. This fact opens the opportunity for ns- 3 users to develop their own consumer functions.

\section{HANDOVER ALGORITHM}

Handover in LTE may utilise the X2 interface between the source eNodeB and the target eNodeB, hence referred to as an X2-based handover. If X2 interface is not available, then S1-based handover may be used instead, but this procedure is not supported by ns-3 at the time of writing.

The source eNodeB is responsible to make a handover decision, i.e., "when" and "where" a handover is necessary to be triggered. This function of eNodeB is also known as the handover algorithm. The 3GPP standard does not provide any specification of such algorithm, thus the exact implementation has to be independently devised by eNodeB vendors. This fact may have contributed to the popularity of handover algorithm as research theme in the LTE field.

Handover algorithms have a very important role in LTE systems. They determine the trade-off between many possible Key Performance Indicators (KPI). For instances, literatures have studied the effect of handover algorithms to signalling overhead [11], outage probability [13, 15], user throughput [17], cell capacity [18], energy efficiency [20], and, in general, service quality. Most of these studies have been conducted by comparing the performance of different

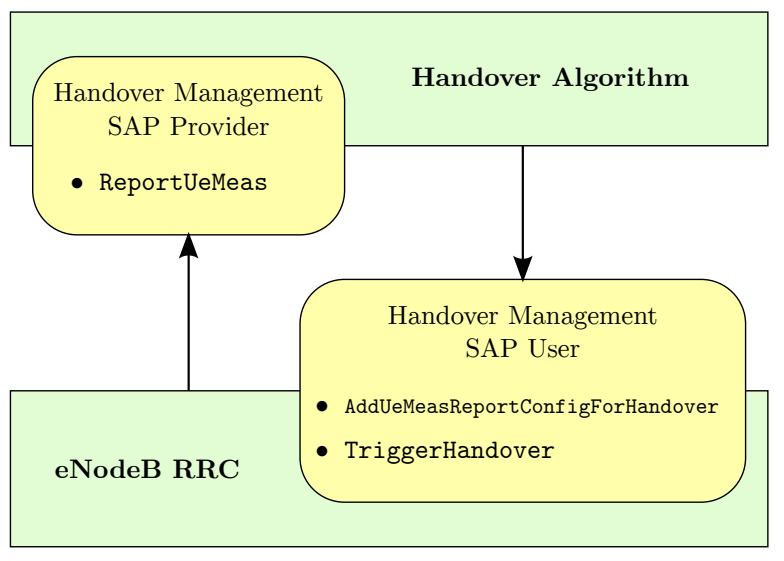

Figure 3: Handover Management SAP

algorithms and/or different parameter configuration using the help of simulations. The extension proposed in this section aims to enable researchers to perform similar studies using ns-3 as the simulator.

\subsection{Modelling}

We redesigned the handover algorithm models in ns-3 as consumers of UE measurements. The advantage of this approach is the possibility of ns-3 users in general to develop their own handover algorithms in a modular fashion as expected by Objective 2 . In practice, the user is expected to write a child class of the LteHandoverAlgorithm abstract class and utilise the common interface with eNodeB RRC, i.e., the Handover Management SAP (Service Access Point).

Similar to the other interfaces in ns-3 LTE module which use the SAP framework, the Handover Management SAP comprises a "provider" part and a "user" part. As illustrated in Figure 3, the "provider" part is used by the eNodeB RRC instance to send messages to the handover algorithm instance. This part contains the ReportUeMeas method, which is used to provide measurement reports produced by the UE measurement function. Hence, it relates to the measurement reporting interface of a consumer of UE measurements.

The "user" part is dedicated for sending messages on the other direction. The first method of this part is the AddUeMeasReportConfigForHandover interface, which allows the handover algorithm instance to perform measurement configuration. Finally, the handover algorithm implementation may invoke the second method, named TriggerHandover, to inform the eNodeB RRC instance that a handover decision has been made.

In addition, the extension includes 3 built-in implementations of handover algorithm, developed using the interfaces explained above. These are the A2-A4-RSRQ, the strongest cell, and the no-op handover algorithm. Each of these algorithms is described in each of the following subsections.

\subsection{A2-A4-RSRQ Handover Algorithm}

Before the above handover algorithm design is in place, ns-3.18 had a working handover algorithm [9] statically embedded inside eNodeB RRC. We converted this algorithm to follow the new design, resulting in a separate consumer entity which we named the A2-A4-RSRQ handover algorithm. 
As the name implies, the algorithm utilises the RSRQ measurements acquired from Event A2 and Event A4. The algorithm is explained in the following.

Let $u$ be the UE currently considered for a handover. Then let $n$ be the neighbour cell which shows the best RSRQ among all neighbour cells detected by UE $u$. In other words:

$$
n=\underset{c}{\arg \max } \operatorname{RSRQ}(u, c)
$$

where $\operatorname{RSRQ}(u, c)$ is the measured RSRQ by UE $u$ of a detected neighbour cell $c$. Then a handover is triggered by the A2-A4-RSRQ handover algorithm on UE $u$ to cell $n$ if and only if the following two inequalities are both true:

$$
\begin{gathered}
\operatorname{RSRQ}(u, s)<\text { Thresh } \\
\operatorname{RSRQ}(u, n)-\operatorname{RSRQ}(u, s) \geq \text { Off }
\end{gathered}
$$

Here, $s$ indicates the serving cell and Thresh is the threshold parameter used by Event A2. Hence, it should be apparent that Inequality (1) is wholly computable by Event A2. The variable $\operatorname{RSRQ}(u, n)$ in Inequality $(2)$, on the other hand, is acquired from Event A4. Lastly, the constant Off enforces a minimum difference of RSRQ that must be surpassed before a handover could happen.

The constants used by the algorithm are configurable from user's simulation programme. The ServingCellThreshold attribute configures the Thresh constant. Another attribute, NeighbourCelloffset, configures the Off constant. They are expressed in 3GPP range unit [3]. For example, the default value of Thresh is 30, which is equivalent to an RSRQ of $-5 \mathrm{~dB}$.

\subsection{Strongest Cell Handover Algorithm}

The second built-in handover algorithm is the strongest cell handover algorithm. It has also been known elsewhere as the traditional power budget (PBGT) handover algorithm. Its concept has been around in various cellular network technologies since at least the late nineties [19]. A more recent instance would be the $3 \mathrm{GPP}$ recommendation for the preceding third generation UMTS standard [1].

The main idea of the algorithm is to provide the best signal strength for each UE. To achieve this, the algorithm triggers a handover on a UE as soon as another cell with a stronger RSRP exists. Hence, the condition for a handover trigger in the strongest cell handover algorithm can be expressed as follows [8]:

$$
\operatorname{RSRP}(u, n)>\operatorname{RSRP}(u, s)+H y s
$$

In order to mitigate possible false alarms caused by channel fading, the algorithm employs the hysteresis parameter Hys and the time-to-trigger parameter. Hys ensures that the neighbour cell is stronger by at least a certain decibels of offset. Then the time-to-trigger parameter enforces that Inequality (3) must be consistently true for at least a certain duration of time before the handover can be triggered.

Inspired by [10], we modelled the above behaviour by using Event A3 and its hysteresis and time-to-trigger parameters. These sub-features were part of the extension to the $\mathrm{UE}$ measurements function described in the previous section. As an example, Figure 4 depicts the trace of RSRP measurements taken from a UE in an ns-3 simulation. The simulation is based on a slightly modified version of ns-3's Ite-x2-handover-measures example script, where a UE is

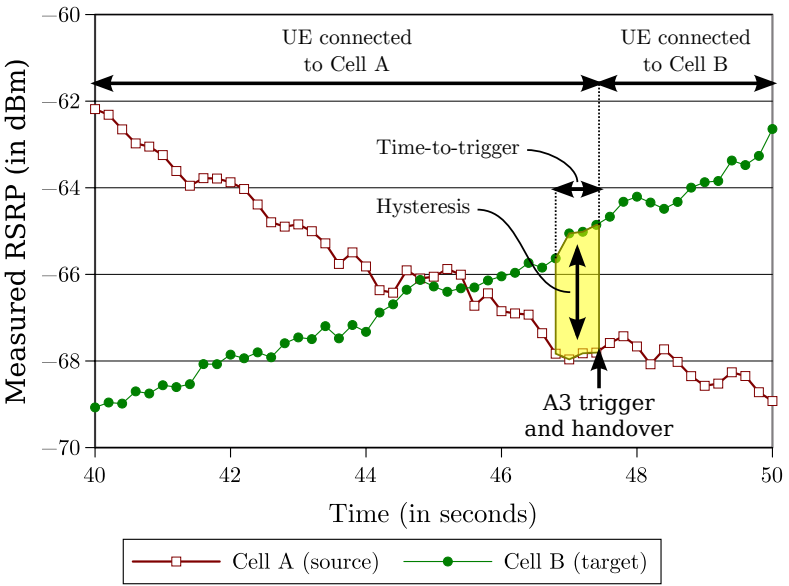

Figure 4: Strongest cell handover algorithm

moving across two neighbouring cells, cell $\mathrm{A}$ and cell $\mathrm{B}$, in a constant speed of $60 \mathrm{~km} / \mathrm{h}$. Notice that the handover trigger (i.e., Event A3) is delayed by a hysteresis of $2 \mathrm{~dB}$ and a time-to-trigger of $640 \mathrm{~ms}$.

The values of hysteresis and time-to-trigger parameters are constant throughout the simulation. User's simulation programme may set the values of these parameters through the ns-3 attribute system. Studies have shown that higher values of hysteresis [12] and time-to-trigger [14] reduce the number of unnecessary handovers, e.g., ping-pong handovers.

\subsection{No-Op Handover Algorithm}

The last built-in handover algorithm is the no-op handover algorithm, which is basically an algorithm which never triggers any handover. Because of this, the algorithm does not require any measurement configuration from the eNodeB RRC instance it is attached to. This algorithm may be used by ns- 3 users to disable handovers from being automatically triggered in their simulations. Moreover, ns-3 users may find the algorithm's source code useful as a skeleton to design and implement their own custom handover algorithms.

\section{INITIAL CELL SELECTION}

Recall that Objective 3 aims to provide an automatic means for UE to attach to the network. In other words, upon start-up, a UE shall be able to detect the surrounding cells, select the best one, and camp on it.

This activity is related to the user mobility function in Idle mode. In 3GPP standard, it consists of 3 related procedures: cell search, cell selection, and cell re-selection [6]. Cell search is a physical layer procedure to detect surrounding cells. Then in cell selection, the UE uses cell measurements and system information to determine which cell it will camp on. Finally, cell re-selection is performed when the UE has already camped on a cell but then it discovers that another cell has become a better candidate for camping. Thus, cell re-selection ensures that the UE is always camped on the "best" cell in terms of received power.

Note that Objective 3 is already accomplished by cell search and cell selection procedures. After these procedures are completed, the UE immediately switches to Connected mode. This approach eliminates the need for subsequent 


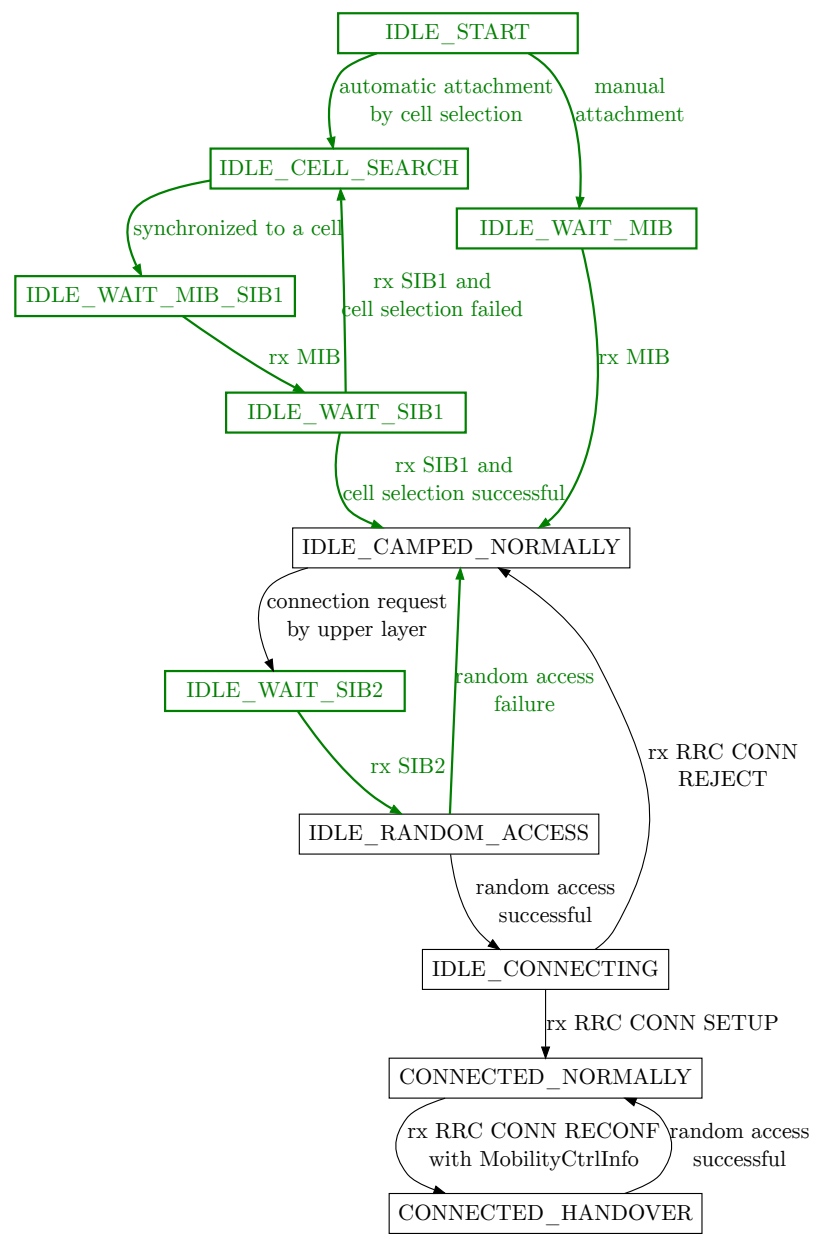

Figure 5: Modified UE RRC state model in ns-3

user mobility in Idle mode. Because of this reason, cell reselection is not considered in this paper.

This section describes the implementation of the initial cell selection procedure in ns- 3 . The word "initial" comes from the fact that the cell selection is performed by the $\mathrm{UE}$ for the very first time. The selection is based on UE measurements information and cell selection criteria. As an alternative to initial cell selection, 3GPP allows the use of stored information [6], speeding up the process, but its realisation in ns- 3 is beyond the scope of this paper.

\subsection{UE RRC State Model}

To accommodate the new initial cell selection procedure in ns-3, we modified the UE RRC state model. The changes are depicted in green colour in Figure 5. Notice the IDLE and CONNECTED prefixes in front of each of the state names, indicating Idle and Connected modes.

The manual attachment branch comes from the existing ns-3 models and is left intact in the modified models. To enter the newly implemented branch, i.e., the automatic attachment branch, the UE NAS entity must invoke the StartCellSearch function. From the perspective of ns-3 users in general, the manual automatic branch can be invoked by using the Attach (ueDevices, enbDevice) method of the LteHelper class, while the automatic branch can be be invoked by using the Attach (ueDevices) method (only one argument) of the same class.

\subsection{Cell Search}

The implemented initial cell selection procedure works as follows. When the ns-3 simulation starts, every UE begins at the IDLE_START state, regardless of the selected attachment branch. If the simulation programme has selected for the UE the automatic attachment branch as described in the previous subsection, the UE invokes an initial cell selection procedure and transitions to the IDLE_CELL_SEARCH state.

After that, the cell search sub-procedure follows immediately. During the course of cell search, the UE measures the RSRP of all surrounding cells. After the measurement period of $200 \mathrm{~ms}$ [3] has elapsed, the UE chooses the strongest cell among the detected cells and then attempts to synchronise to the chosen cell. Let $c$ be the reference to this cell.

\subsection{System Information Acquisition}

When the synchronisation is completed, the UE moves on to the IDLE_WAIT_MIB_SIB1 state. In this state, the UE simply waits for system information messages to be received from cell $c$. These messages are Master Information Block (MIB) and System Information Block Type 1 (SIB1).

The transmission of these messages by the eNodeB follows a fixed schedule [7]. MIB is transmitted at the beginning of every radio frame (i.e., every $10 \mathrm{~ms}$ ). It contains parameters related to physical layer, such as the system operating bandwidth. SIB1 is transmitted at the middle of every second radio frame (i.e., every $20 \mathrm{~ms}$ ) and contains information regarding network attachment.

\subsection{Cell Selection Evaluation}

After both MIB and SIB1 have been received, the UE evaluates a simplified version of the standard cell selection criteria [6] on cell $c$, as described in the following.

\subsubsection{Rx Level Criterion}

The first criterion used in the evaluation is the $\mathrm{Rx}$ level criterion, as outlined below:

$$
Q_{\text {rxlevmeas }}-Q_{\text {rxlevmin }}>0
$$

Here, $Q_{\text {rxlevmeas }}$ is the measured RSRP (without layer 3 filtering) of cell $c$ and $Q_{\text {rxlevmin }}$ is a constant determined by SIB1.

\subsubsection{CSG Criterion}

The second criterion is based on Closed Subscriber Group (CSG) information. This criterion is only evaluated if SIB1 indicated "true" for the CSG indication field. In this case, the CSG identity of cell $c$ (also provided as one of the fields in SIB1) must match with the CSG identity of the UE.

Note that a real UE device is not limited to only one CSG identity, but may be associated to multiple identities. These identities are collectively referred to as the $C S G$ whitelist.

\subsubsection{Evaluation Outcome}

If both of the above criteria are passed, cell $c$ is deemed as suitable. Then the UE camps on cell $c$, changing the UE's state to IDLE_CAMPED_NORMALLY. The initial cell selection procedure is therefore completed successfully at this point. 
A more elaborate scenario occurs when cell $c$ does not fulfil the CSG criterion. In this case, cell $c$ shall be marked as acceptable and the UE shall neither camp on nor connect to cell $c$. The UE shall retry the cell search procedure and synchronise to the strongest non-acceptable cell (i.e., cell which has not been marked as acceptable). This procedure shall repeat until the UE finds a suitable cell to camp on.

In contrast, the $3 \mathrm{GPP}$ standard [6] allows UE to temporarily camp on an acceptable cell and receive limited service. This feature is related to the Any Cell Selection state, which is outside the scope of this paper.

\subsection{Next Step after Initial Cell Selection}

By completing an initial cell selection procedure, the UE has successfully entered the Idle mode. In a real LTE system, the UE would indefinitely stay in this mode until an actual connection is established. However, given the fact that other features of Idle mode have not been supported yet in ns-3, it is unlikely that one can gain sufficient scientific value from simulating a $\mathrm{UE}$ in Idle mode at this moment. Because of this reason, the automatic attachment procedure includes a call to the Connect function of the UE NAS entity. This method will cause the UE to proceed towards Connected mode as soon as it reaches the IDLE_CAMPED_NORMALLY state. In other words, the UE will immediately switch to Connected mode after the initial cell selection procedure is completed.

\section{TESTING AND VALIDATION}

In order to validate the developed models, we have extended the existing ns-3 test suites from [9] and also created new test suites to stretch the overall test coverage. This section describes these test suites.

\subsection{UE Measurements Tests}

The UE measurements function of [9] had an existing test suite named lte-ue-measurements. Its main focus was to verify the correctness of the RSRP and RSRQ measurements as reported by the UE and received by the eNodeB.

To complement this test suite, we created 3 new test suites which were focused on verifying the timing aspect of UE measurements. In other words, the areas covered by these test suites are the measurement configuration and measurement reporting functions. There are two types of test scenarios, namely the piecewise and the handover scenario.

\subsubsection{Piecewise Scenario}

Event-based measurement report triggering is determined by the entering condition and the leaving condition of the corresponding event [7]. Once the event's entering condition is fulfilled, UE begins sending measurement report periodically to the serving eNodeB. Then the periodical reporting shall stop once the event's leaving condition is fulfilled. If the time-to-trigger parameter is configured for the event, then the above conditions must be applicable during the timeto-trigger duration before the periodical reporting can be started or stopped. Entering conditions are logically similar to the event descriptions from Table 1, while leaving conditions are similar to the inverse of the event descriptions.

The piecewise scenario consists of 4 pre-determined "spots" in the simulation environment and a single UE which moves between these spots in a fixed pattern. The movement fluc-

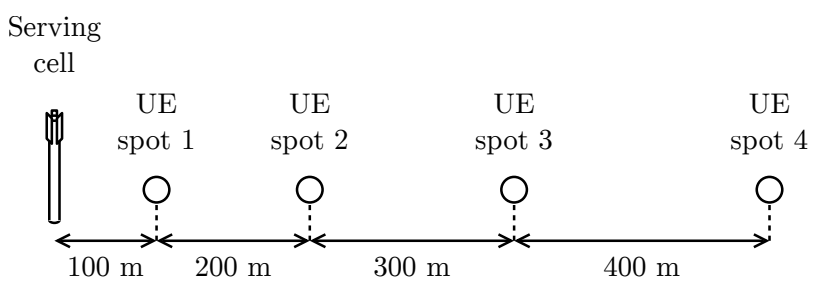

Figure 6: Pre-defined UE measurement spots in the first piecewise scenario

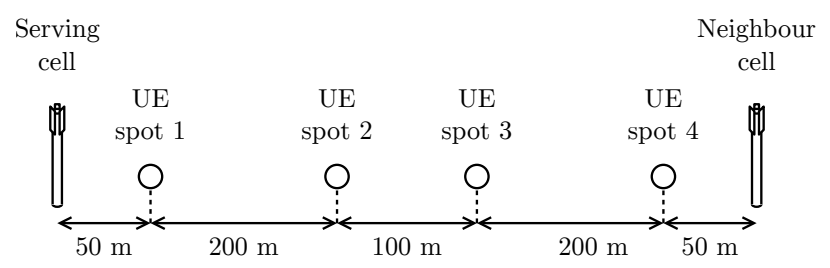

Figure 7: Pre-defined UE measurement spots in the second piecewise scenario

tuates the UE's measurement results, and thus possibly invoking the entering and/or leaving conditions.

There are two types of piecewise scenarios. The first scenario, depicted in Figure 6, consists of one eNodeB. The second scenario, depicted in Figure 7, has an additional eNodeB which plays the role of a neighbour cell, and thus is useful for testing event-based triggers which are dependent on measurements from neighbour cells. The no-op handover algorithm (Subsection 3.4) is used in this scenario to prevent the UE from being handed over to the neighbour cell.

The first piecewise scenario is implemented in the lte-uemeasurements-piecewise-1 test suite. The second piecewise scenario is implemented in the lte-ue-measurementspiecewise-2 test suite. These test suites repeatedly execute the scenarios using different settings, covering all reporting triggers from Table 1 and their underlying parameters in different level of values. The tests verify that measurement reports are only sent at the prescribed times during the test simulation and that they contain the correct level of RSRP (RSRQ is not verified at the moment).

\subsubsection{Handover Scenario}

Measurement configuration is modelled as a cell-specific configuration. Therefore, it is possible that two cells have different measurement configuration. When a UE performs a handover between these two cells, the UE's active measurement configuration must be updated to reflect the measurement configuration of the target cell. This requirement is verified by the handover scenario, sketched in Figure 8.

This concept is realised by the Ite-ue-measurementshandover test suite. The source eNodeB and target eNodeB in this scenario are configured with different measurement configuration. The UE is configured as static (i.e., not moving), so the handover is triggered manually by the test script at the middle of the test simulation. As in the previously described test suites, the scenario is repeatedly executed using different measurements configuration.

Note that this test suite does not verify the handover procedure. Handover in ns-3 is verified by another group of test suites, which are described in the following subsection. 


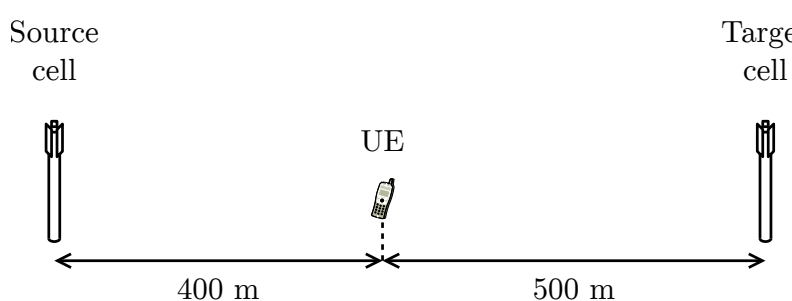

Figure 8: Handover scenario for measurements test

\subsection{Handover Tests}

The validation of handover reused an existing ns-3 test suite named 1te-x2-handover-measures. The test suite simulates a UE moving from the coverage area of one cell to the coverage area of another cell, and then verifies that a handover occurred and completed successfully. It is simply assumed that the CONNECTED_NORMALLY state on the UE side indicates a successful handover. More details on the test suite has been provided in [9].

On top of that, we developed a new test suite which is more closely related to the handover algorithm functionality. The 1te-handover-target test suite reproduces a situation where the algorithm is faced with an imminent handover and 3-5 possible candidates of target cell. One of the scenarios is illustrated in Figure 9. The test suite verifies that the algorithm is able to sort these candidates and choose the most desirable one as the target cell.

Both A2-A4-RSRQ and strongest cell handover algorithms are tested in both of the above test suites.

\subsection{Initial Cell Selection Test}

A newly developed test suite named lte-cell-selection covers the initial cell selection procedure. It verifies whether UE may attach and connect to the network by utilising the procedure. This is specifically done by checking the correctness of the cell selected by the procedure and then verifying that the UE's final state is CONNECTED_NORMALLY.

The test suite takes into account several scenarios of cell selection. As in the previous section, let $c$ be the first cell found by the cell search procedure. The most probable type of scenario is the case where cell $c$ is a suitable cell, i.e., cell $c$ successfully passes the cell selection evaluation. In this case, the UE shall be able to camp on and connect to cell $c$ within a short period of time. In another type of scenario, cell $c$ is an acceptable cell; therefore the UE shall be able to repeat the procedure on another cell until it finds a suitable cell. All of the tested scenarios are depicted in Figure 10.

\section{CONCLUSIONS}

In this paper, we have described the implementation of simulation models to improve the user mobility feature of ns-3 LTE simulator. The improvement to the user mobility function in Connected mode consisted of extensions to the UE measurements and the handover algorithm functions. The strong point of the design was the high degree of configurability through the use of ns-3 attribute system and custom handover algorithm. On the other hand, user mobility function in Idle mode has been improved by introducing an automatic method of network attachment. We modelled an initial cell selection procedure to achieve this. Finally, we presented a set of tests used to validate the implementation.
Time: $+0.0 \mathrm{~s}$

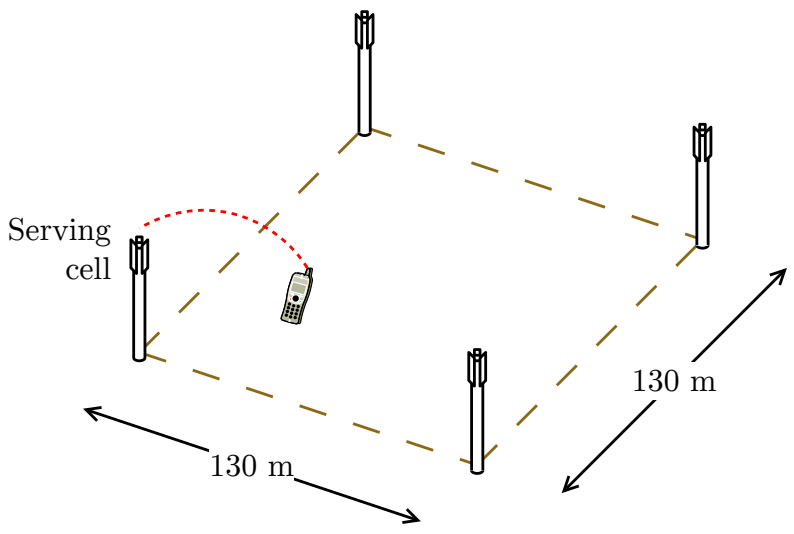

Time: $+0.5 \mathrm{~s}$

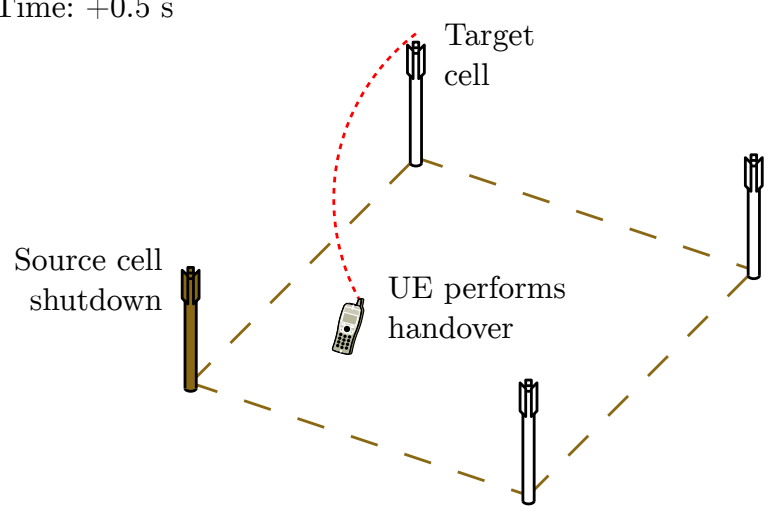

Figure 9: Handover algorithm test scenario

CSG cell/member $\quad . . \cdots \cdots$ Successful cell selection ,- - Failed cell selection

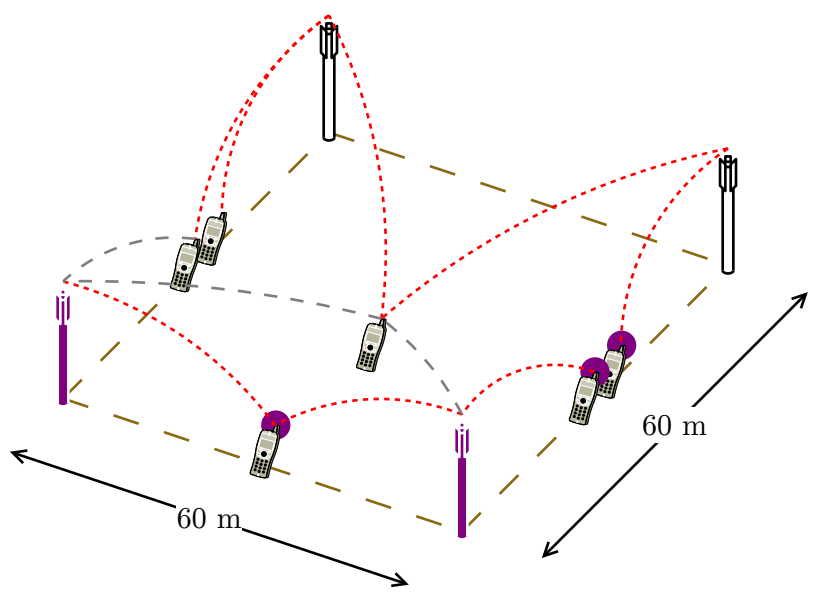

Figure 10: Initial cell selection test scenario 
We note that the limited support of Idle mode has restricted further development of LTE mobility features in ns-3. An interesting follow-up of this paper would be to implement the remaining Idle mode procedures, which may open the opportunity to conduct more advanced LTE mobility studies using ns-3. Such improvement may include features such as Radio Link Failures (RLF), handover failures, and cell re-selection. We also note that the CSG criterion should also be replicated to the handover procedure, but this has also been left as a future work.

The work described in this paper has undergone several rounds of review by the ns- 3 project. There were two feature specific reviews, ${ }^{2}{ }^{3}$ a mid-term review, ${ }^{4}$ and the final review. 5 At last, the final set of deliverables was merged with the main ns-3 distribution and was released in December 2013 under the ns-3.19 release. The source code is publicly available for download from ns-3 website at www.nsnam.org under the GNU General Public License v2. For instructions on using the models described in this paper, users may refer to the accompanying documentation in Model Library > LTE Module > User Documentation, specifically under the Network Attachment, Configure UE Measurements, X2-Based Handover, and Handover Simulation Campaign sections.

\section{ACKNOWLEDGEMENTS}

The work presented in this paper was generously funded by the Google Summer of Code 2013 programme. The work done at the Centre Tecnològic de Telecomunicacions de Catalunya was also partially funded by the Spanish Ministry of Science and Innovation through the project SYMBIOSIS (TEC2011-29700-C02-01) and by the Catalan Regional Government under grant 2009SGR-940.

In addition to the above, the authors would also like to sincerely thank Tom Henderson, Brian Swenson, and Lalith Suresh of ns-3 project for their constructive feedback and support. Another bunch of thanks goes to Jani Puttonen, Niko Kolehmäinen, and Janne Kurjenniemi of Magister Solutions for the advices and useful discussions during the course of the project.

\section{REFERENCES}

[1] 3GPP. TR 25.922; UMTS; radio resource management strategies, 2007.

[2] 3GPP. TR 25.913; UMTS; LTE; requirements for E-UTRA and E-UTRAN, 2010.

[3] 3GPP. TS 36.133; LTE; E-UTRA; requirements for support of radio resource management, 2013.

[4] 3GPP. TS 36.214; LTE; E-UTRA; physical layer; measurements, 2013.

[5] 3GPP. TS 36.300; LTE; E-UTRA and E-UTRAN; overall description; stage 2, 2013.

[6] 3GPP. TS 36.304; LTE; E-UTRA; user equipment (UE) procedures in idle mode, 2013.

[7] 3GPP. TS 36.331; LTE; E-UTRA; radio resource control (RRC); protocol specification, 2013.

\footnotetext{
${ }^{2}$ https://codereview . appspot.com/11061044/

${ }^{3}$ https://codereview . appspot . com/12657043/

${ }^{4}$ https://codereview. appspot.com/11670043/

${ }^{5}$ https://codereview . appspot.com/13720052/
}

[8] M. Anas, F. Calabrese, P. Mogensen, C. Rosa, and K. Pedersen. Performance evaluation of received signal strength based hard handover for UTRAN LTE. In Vehicular Technology Conference (VTC 200\%-Spring), 65th IEEE, pages 1046-1050, 2007.

[9] N. Baldo, M. Requena-Esteso, M. Miozzo, and R. Kwan. An open source model for the simulation of LTE handover scenarios and algorithms in ns-3. In Modeling, Analysis and Simulation of Wireless and Mobile Systems (MSWiM), 16th ACM International Conference on, pages 289-298, 2013.

[10] K. Dimou, M. Wang, Y. Yang, M. Kazmi, A. Larmo, J. Pettersson, W. Muller, and Y. Timner. Handover within 3GPP LTE: Design principles and performance. In Vehicular Technology Conference (VTC 2009-Fall), 70th IEEE, pages 1-5, 2009.

[11] B. Jeong, S. Shin, I. Jang, N. W. Sung, and H. Yoon. A smart handover decision algorithm using location prediction for hierarchical macro/femto-cell networks. In Vehicular Technology Conference (VTC 2011-Fall), 74th IEEE, pages 1-5, 2011.

[12] K. Kitagawa, T. Komine, T. Yamamoto, and S. Konishi. A handover optimization algorithm with mobility robustness for LTE systems. In Personal Indoor and Mobile Radio Communications (PIMRC), IEEE 22nd International Symposium on, pages 1647-1651, 2011.

[13] D.-W. Lee, G.-T. Gil, and D.-H. Kim. A cost-based adaptive handover hysteresis scheme to minimize the handover failure rate in $3 \mathrm{GPP}$ LTE system. Wireless Communications and Networking, EURASIP Journal on, 2010(1):750173, 2010.

[14] Y. Lee, B. Shin, J. Lim, and D. Hong. Effects of time-to-trigger parameter on handover performance in SON-based LTE systems. In Communications (APCC), 16th Asia-Pacific Conference on, pages 492-496, 2010.

[15] P. Legg, G. Hui, and J. Johansson. A simulation study of LTE intra-frequency handover performance. In Vehicular Technology Conference (VTC 2010-Fall), 72nd IEEE, pages 1-5, 2010.

[16] P. Lescuyer and T. Lucidarme. Evolved Packet System (EPS): The LTE and SAE Evolution of $3 G$ UMTS. Wiley, 2008.

[17] C.-C. Lin, K. Sandrasegaran, H. Ramli, R. Basukala, R. Patachaianand, L. Chen, and T. Afrin. Optimization of handover algorithms in 3GPP long term evolution system. In Modeling, Simulation and Applied Optimization (ICMSAO), 4th International Conference on, pages 1-5, 2011.

[18] A. Lobinger, S. Stefanski, T. Jansen, and I. Balan. Load balancing in downlink LTE self-optimizing networks. In Vehicular Technology Conference (VTC 2010-Spring), 71st IEEE, pages 1-5, 2010.

[19] G. Pollini. Trends in handover design. Communications Magazine, IEEE, 34(3):82-90, 1996.

[20] D. Xenakis, N. Passas, and C. Verikoukis. A novel handover decision policy for reducing power transmissions in the two-tier LTE network. In Communications (ICC), IEEE International Conference on, pages 1352-1356, 2012. 